\title{
Chronic Contained Rupture of an Abdominal Aortic Aneurysm: From Diagnosis to Endovascular Resolution
}

\author{
Roberto Gandini · Marcello Chiocchi · Luciano Maresca · Vincenzo Pipitone • \\ Massimo Messina - Giovanni Simonetti
}

Received: 28 January 2007 / Accepted: 25 July 2007/Published online: 21 August 2007

(C) Springer Science+Business Media, LLC 2007

\begin{abstract}
A male patient, 69 years old, presented with fever, leucocytosis, and persistent low back pain; he also had an abdominal aortic aneurysm (AAA), as previously diagnosed by Doppler UltraSound (US), and was admitted to our hospital. On multislice computed tomography (msCT), a large abdominal mass having no definite border and involving the aorta and both of the psoas muscles was seen. This mass involved the forth-lumbar vertebra with lysis, thus simulating AAA rupture into a paraspinal collection; it was initially considered a paraspinal abscess. After magnetic resonance imaging examination and culture of the fluid aspirated from the mass, no infective organisms were found; therefore, a diagnosisof chronically contained AAA rupture was made, and an aortic endoprosthesis was subsequently implanted. The patient was discharged with decreased lumbar pain. At 12-month follow-up, no evidence of leakage was observed. To our knowledge, this is the first case of endoprosthesis implantation in a patient, who was a poor candidate for surgical intervention due to renal failure, leucocytosis and high fever, having a chronically contained AAA ruptured simulatingspodilodiscitis abscess. Appropriate diagnosis and therapy resolved potentially crippling pathology and avoided surgical graftrelated complications.
\end{abstract}

R. Gandini · M. Chiocchi - L. Maresca · V. Pipitone ·

M. Messina $\cdot$ G. Simonetti

Department of Diagnostic and Molecular Imaging,

Interventional Radiology, Nuclear Medicine and Radiation

Therapy, University of Rome "Tor Vergata, 81 Oxford Street,

Rome 00133, Italy

R. Gandini $(\bowtie)$

Department of Radiology, University of Rome " Tor Vergata ,",

81 Oxford street, Rome 00133, Italy

e-mail: marcello.chiocchi@fastwebnet.it
Keywords Abdominal aortic aneurysm . Endoprosthesis implantation - Vertebral erosion

Lumbar pain is a nonspecific sign of disease. Among the factors that determine its onset, the presence of a chronic broken aneurysm is an uncommon but valid indication for surgery. Choplin et al. [1] described a ruptured abdominal aortic aneurysm (AAA) simulating pyogenic vertebral spondylitis with vertebral erosion in 1982. Others investigators [2-5] reported chronically contained AAA rupture as early as 1986. In our case, Multislice computed tomography (msCT) and magnetic resonance imaging (MRI) helped differentiate the cause of the vertebral lysis as a chronically contained AAA rupture rather than osteomyelitis. Diagnostic imaging, integrated with endovascular approach, correctly identified this pathology and allowed us to resolve it in a less-invasive manner.

\section{Case Report}

A 69-year-old man with a 3-year clinical history of endstage renal failure and dialysis and an AAA, as previously diagnosed by Doppler UltraSound (US), located below the origin of the renal artery. He presented at our hospital with fever $\left(38.5^{\circ} \mathrm{C}\right.$, duration 1 week), leucocytosis $(12,000 / \mathrm{fll})$, persistent low back pain transmitted to the left gluteuscrural region that did not respond to corticosteroids and nonsteroidal anti-inflammatory drugs.

A msCT scan was performed (Light Speed Plus [4 rows]; General Electric) (Fig. 1) to exclude aneurismal fissuration; this confirmed an AAA (7 cm in diameter) located just $1.5 \mathrm{~cm}$ below the renal arteries and extending to the iliac bifurcation. Apparent maximum diameter of the lesion reached $>17 \mathrm{~cm}$ because of its extension along the 
paravertebral spaces into a mass with soft-tissue density. This mass, extending to the common iliac arteries, presented intralesional air bubbles and a thick, calcified peripheral ring and infiltrated the ileo-psoas muscles, with lysis of the fourth lumbar vertebra. No narrowing of the spinal canal was present.

This msCT finding, because of the presence of both intralesional air with a ring enhancement and extension into the left pelvis, showed what was suspected to be osteomyelytic spondylolysis with paraspinal abscess that had probably fistulised into the aorta.

To confirm the presence of nerve involvement and ensure the inflammatory origin of the mass, magnetic resonance angiogram (MRA) (1.5T Achieva Philips Medical System; Best, The Netherlands) was performed by means of spiral sagittal TI-T2-weighted coronal gradient-echo (GE) sequences and axial and sagittal contrast-enhanced T1-weighted sequences.

The lesion showed central isointense and peripheral hyperintense signals compared with muscle intensity on Tlweighted images, and it showed central hyperintensity and peripheral heterogeneous signal intensity on T2-weighted images. No enhancement was detected in or around the lesion.

Signal intensity alteration of L4, with lytic aspect of the soma and poor discal involvement, were noted (Figs. 2A and $2 \mathrm{~B}$ ). Because there was no discernible plane between the dilated aorta and the mass, it was thought to be a pseudo aneurysm of the aorta eroding into the vertebra; moreover, the results of msCT-guided needle aspiration using a 19G 20-cm needle with a 5F (outer diameter) sheath (TLA needle sheath; Boston Scientific, Watertown, MA) in the site of the psoas fluid collection demonstrated dense, brown fluid with some particles of blood clot, but no pathologic organisms grew on cultures.

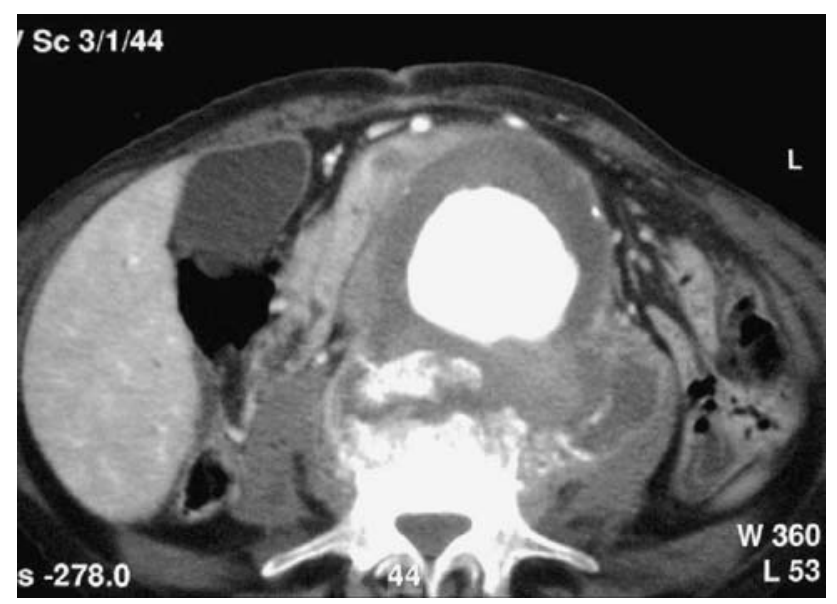

Fig. 1 Abdominal multislice computed tomography (msCT) msCT image shows an infrarenal AAA rupture with vertebral body erosion
After conferring with the vascular surgeon and anaesthesiologist, it was decided to perform endovascular exclusion of the aneurysm. Radiologists planned the endovascular treatment and the choice of type and dimension of the device based on msCT findings and intraprocedural angiography.

A team of two vascular surgeons and two interventional radiologists performed the procedure in an Angio-Suite (ARC X12; Philips Medical Systems, Best, The Netherlands). Surgical exposure of both femoral arteries and placement of an aortic endoprosthesis (main body $28-\mathrm{mm}$ diameter, iliac segments $14.5 \mathrm{~mm}$, and length $16 \mathrm{~cm}$ [Excluder; Gore, Flagstaff, AZ]) was accomplished using the left femoral approach, and left hypogastric artery embolisation with five Gianturco coils (Cook, Bloomington, IN) was carried out by the interventional radiologists. A contralateral endoprosthesis (proximal diameter $16 \mathrm{~mm}$, distal diameter $14 \mathrm{~mm}$, and length $12 \mathrm{~cm}$ ) plus an aortic extender (diameter $23 \mathrm{~mm}$ and length $3.3 \mathrm{~cm}$ ) (Excluder) was subsequently positioned in the aneurysmatic right iliac artery. Postprocedural angiography showed exclusion of the aneurysmatic sac, and despite the patient's renal failure,
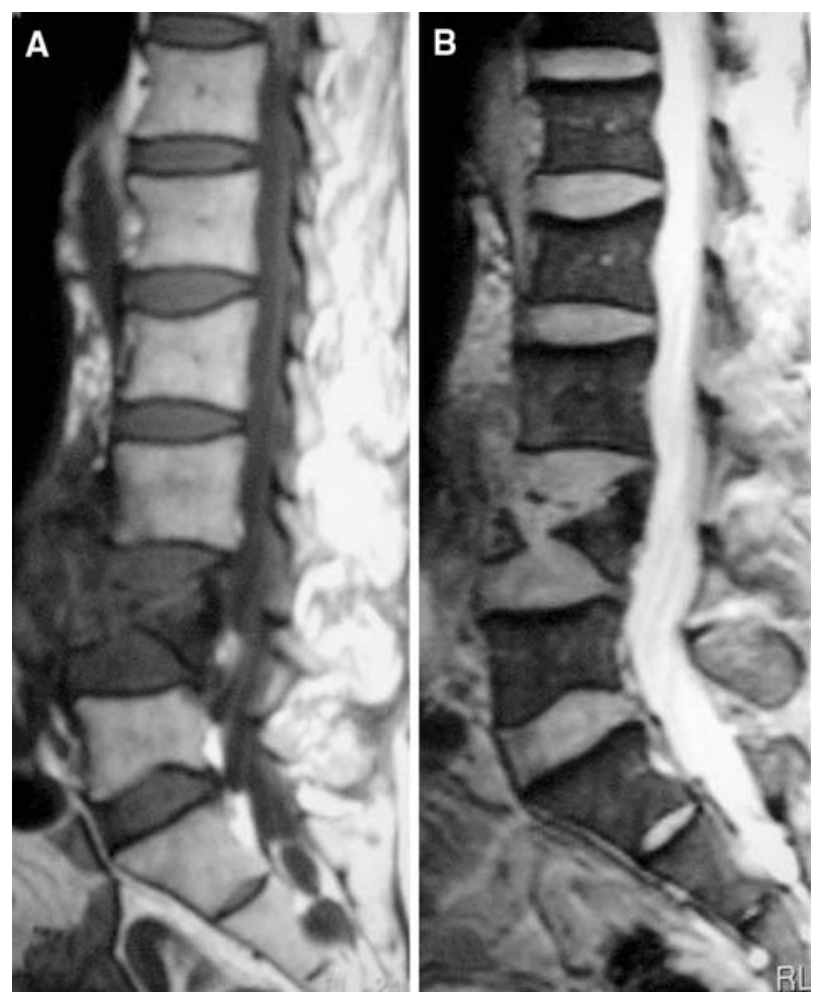

Fig. 2 (A) T1-weighted/TSE (Turbo Spin Echo) sagittal (TE 12, TR 682) and (B) T2-weighted/FFE (Fast Field Echo) sagittal (TE 9.2, TR 450) MRI images of the lumbar spine shows a large defect in the fourth lumbar vertebrae. The anterior margins of the vertebrae are completely eroded by what appears to be a predominantly prevertebral mass. The posterior margin of the aorta is not well depicted on these sequences 


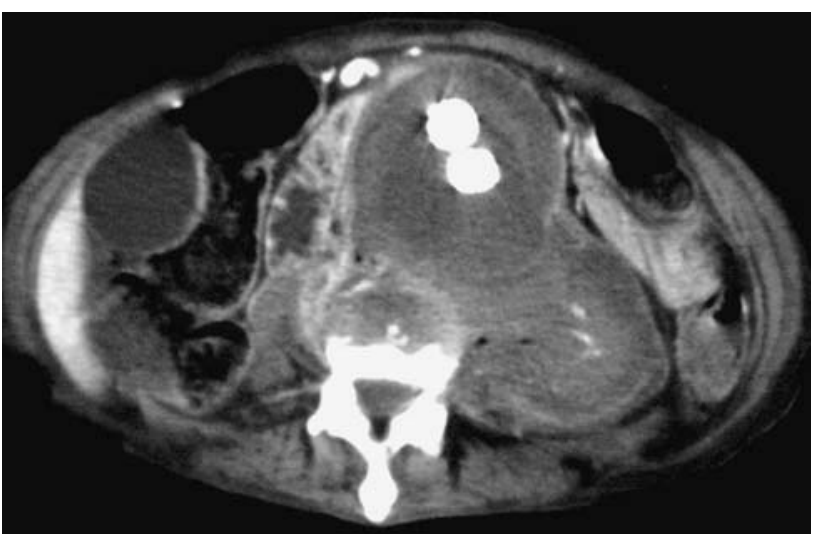

Fig. 3 Abdominal multislice spiral CAT. Abdominal CAT shows total exclusion of the aneurysmatic sac after positioning of the endoprosthesis

there was preservation of renal artery patency and no intraprocedural complications.

Antibiotic therapy with amoxicillin $(2 \mathrm{~g} / \mathrm{d})$ was administrated 1 day previous to and for 5 days after the intervention. Postprocedural therapy was adopted only in this case cause of patient' leucocytosis. The patient was discharged 11 days after in hospital admission with decreased lumbar pain, most of which could be managed with nonsteroidal anti-inflammatory drugs.

No pathologic organisms or infection were noted at the time of the patient's discharge (leucocytes 8000/fll). At 1-, 6-, and 12-month follow-up (Fig. 3), preferred msCT color-Doppler UltraSound and to other x-ray exams, no evidence of leakage nor increase or decrease of the aneurismal diameter was observed; however, the patient still had some residual back pain, caused by excessive bone loss, that was not solvable with vertebroplasty.

\section{Discussion}

Rupture of an AAA is a surgical emergency with a mortality rate, if untreated, of $90 \%$ at 6 weeks and an operative mortality rate of $30 \%$ to $60 \%$ [6-9]. In patients without hypertension [1, 10-12], a sealed aneurism could occur, without any significant change in blood values because of restricted blood loss. These patients are often clinically stable for prolonged periods and are defined as having a chronically contained rupture (also termed "sealed," "spontaneously healed," or "leaking") of the AAA [2, 3, 13].

Sealed rupture of an AAA causing vertebral erosion is rare, and on review of the literature, we found only a few such cases $[1,4,5,10,11,14,15]$. All patients had normal or high blood pressure, and they were hemodynamically stable at initial presentation. The most common presenting

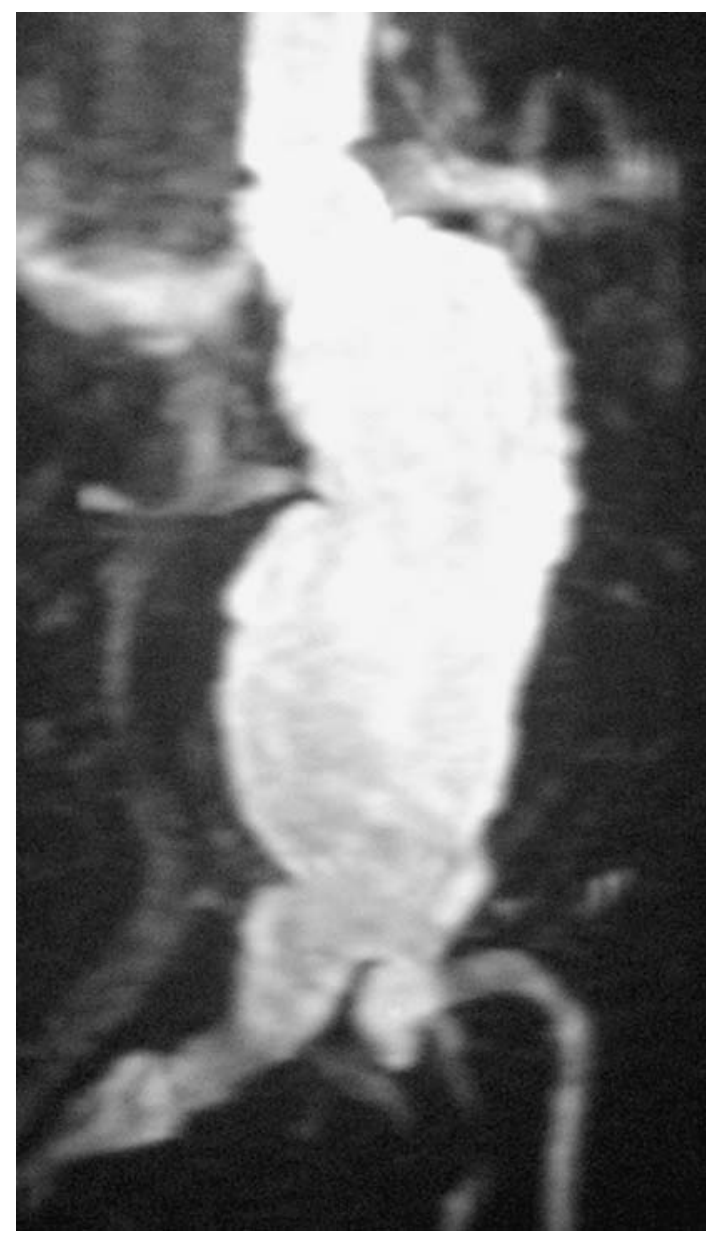

Fig. 4 T1-weighted/FFE/MIP (Maximum Intensity Projection) coronal (TE 1.3, TR 17) MRI images. Angio-MRI coronal-MIP shows a large infrarenal AAA extending to the left and right common iliac arteries

symptom was back pain; the duration of symptoms ranged from 2 weeks to 2 years. The mechanism of chronically contained AAA rupture is not clear. It is believed that in large aneurysms, a strong perianeurysmal reaction subsequently provides high resistance to extravasation of blood [13].

Moreover, the site of sealed AAA rupture has been reported as posterior in $62.5 \%$ of cases, suggesting that vertebrae can tamponade the rupture effectively because of their inherent strength [13]; transmitted arterial pulsation and vertebral compression of 80 to 90 times/min may produce extensive bone destruction with time [13], involving nerve roots or fibers that pass around muscles [7, 15-17]. Erosion caused by aneurysms is usually smooth compared with that caused by pyogenic infection, in which the margins of the lytic process are irregular and poorly defined $[3,18]$.

msCT and MRI are fundamental to accurate and timely diagnosis. They are necessary to assess adequate intervention to avoid rupture into the intraperitoneal or 


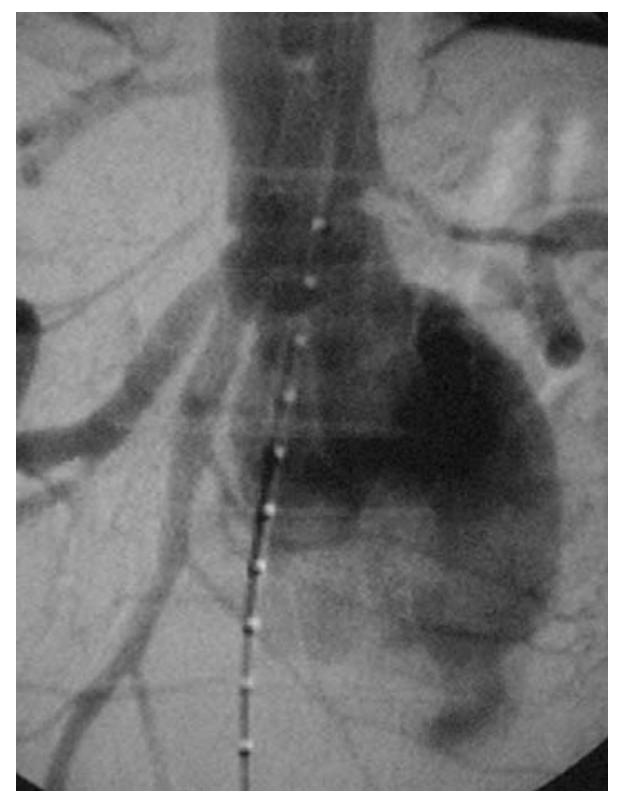

Fig. 5 Diagnostic angiography with centimetred pig-tail confirmed a large infrarenal AAA with a proximal neck $1.5 \mathrm{~cm}$ in length

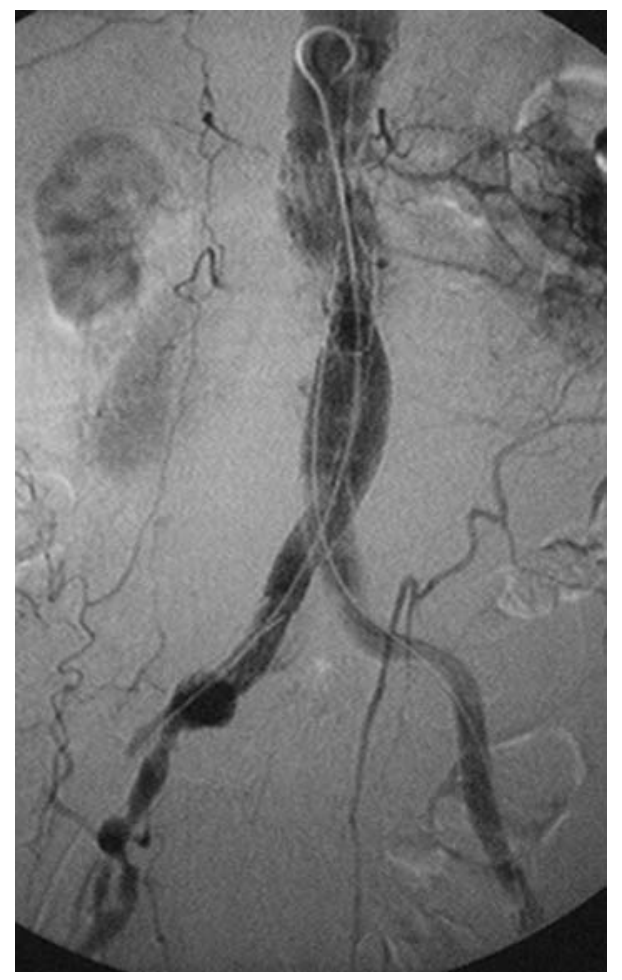

Fig. 6 Angiography after position of the endoprosthesis demonstrates complete exclusion of the aneurysmatic sac

retroperitoneal cavity and the surrounding hollow structures (e.g., veins and bowel). Diagnostic imaging can also be used to accurately distinguish abdominal or back pain attributable to ruptured AAA from other causes [14, 17-23].

Our case report demonstrates how appropriate diagnosis and therapy can resolve crippling pathology and avoid surgical graft-related complications, such as pseudoaneurysm or haemorrhage at the anastomotic site, graft thrombosis, atheroembolism or infection, graft-enteric erosion, and colon ischaemia. A complication rate of $9.4 \%$, especially in a patient such as ours, who was a poor candidate for surgery, has been reported [9, 14, 22].

msCT or MRA, integrated with endovascular therapy, provides an invaluable, less-invasive means by which to depict and exclude the aneurysmal sac; provides prompt repair of this dangerous pathology, which is difficult to identify; and re-establishes an acceptable quality of life for the patient.

To our knowledge, this is the first case of endoprosthesis implantation in a patient, who was a poor candidate for surgical intervention, with chronically contained AAA rupture simulating a spodilodiscitis abscess.

\section{References}

1. Choplin RH, Karstaedt N, Wolfman NT (1982) Ruptured abdominal aortic aneurysm simulating pyogenic vertebral spondylitis. AJR Am J Roentgenol 138:748-750

2. Rosenthal D, Clark MD, Stanton PE, et al. (1986) "Chroniccontained" ruptured abdominal aortic aneurysm: Is it real? J Cardiovasc Surg (Torino) 27:723-724

3. Jones CS, Reilly KM, Dalsing MC, et al. (1986) Chronic contained rupture of abdominal aortic aneurysm. Arch Surg 121:542-545

4. Bansal M, Bansal M, Thukral BB, et al. (2006) Contained rupture of a thoracoabdominal aortic aneurysm presenting as a back mass. J Thorac Imaging 21(3):219-221

5. Saiki M, Urata Y, Katoh I, et al. (2006) Chronic contained rupture of an abdominal aortic aneurysm with vertebral erosion: Report of a case [review]. Ann Thorac Cardiovasc Surg 12(4):300-302

6. Gavant ML, Salazar JE, Ellis J (1986) Case report. Intrarenal rupture of the abdominal aorta: CT features. J Comput Assist Tomogr 4:529-532

7. Darling RC (1970) Ruptured arteriosclerotic abdominal aortic aneurysms. A pathologic and clinical study. Am J Surg 119:402407

8. Dillon M, Cardwell C, Blair PH, et al. (2007) Endovascular treatment for ruptured abdominal aortic aneurysm [review]. Cochrane Database Syst Rev 24(1):CD005261

9. Biancari F, Ylonen K, Anttila V, Juvonen J, Romsi P, Satta J, et al. (2002) Durability of open repair of infrarenal abdominal aortic aneurysm: A 15-year follow-up study. J Vasc Surg 35(1):87-93

10. Prete PE, Thorne RP, Robinson CA (1980) Low-back pain and vertebral erosion due to aortic anastomotic false aneurysm, with documentation by computerized tomography: A case report. J Bone Joint Surg Am 62:126-128

11. Galessiere PF, Downs AR, Greenberg HM (1994) Chronic, contained rupture of aortic aneurysms associated with vertebral erosion. Can J Surg 37:23-28

12. Szilagyi DE (1982) Clinical diagnosis of intact and ruptured abdominal aortic aneurysms. In: Bergan JJ, Yao JST (eds) Aneurysms: Diagnosis and treatment. New York, NY, Grune \& Stratton, pp 205-216

13. Sterpetti AV, Blair EA, Schultz RD, et al. (1990) Sealed rupture of abdominal aortic aneurysms. J Vasc Surg 11:430-435 
14. Kapoor V, Kanal E, Fukui MB (2001) Vertebral mass resulting from a chronic-contained rupture of an abdominal aortic aneurysm repair graft. AJNR Am J Neuroradiol 22(9):17751777

15. Defraigne JO, Sakalihasan N, Lavigne JP, et al. (2001) Chronic rupture of abdominal aortic aneurysm manifesting as crural neuropathy [review]. Ann Vasc Surg 15(3):405-411

16. Higgins R, Peitzman AB, Reidy M, et al. (1998) Chronic contained rupture of an abdominal aortic aneurysm presenting as a lower extremity neuropathy. Ann Emerg Med 17:284-287

17. Ando M, Igari T, Yokoyama H, et al. (2003) CT features of chronic contained rupture of an abdominal aortic aneurysm. Ann Thorac Cardiovasc Surg 9:274-278

18. Jacquot JM, Strubel D, Joyeux A, et al. (1996) Sealed rupture of an abdominal aortic aneurysm with chronic vertebral destruction as the first manifestation. Contribution of computed tomography to the diagnosis. Rev Rhum Engl Ed 63(5):377-379
19. William LR, Flinn WR, Yao JST (1985) Extended use of computed tomography in the management of complex aortic problem: a learning experience. J Vasc Surg 4:264-271

20. Senapatti A, Hurst PAE, Thomas IL (1986) Differentiation of ruptured aortic aneurysm from acute expansion by computerized tomography. J Cardiovasc Surg (Torino) 27:719-722

21. Johnson WC, Gale ME, Gerzol SG (1986) The role of computed tomography in symptomatic aortic aneurysms. Surg Gynecol Obstet 162:49-53

22. Hallet JW Jr, Marshall DM, Petterson TM, et al. (1997) Graftrelated complications after abdominal aortic aneurysm repair: Reassurance from a 36-year population-based experience. J Vasc Surg 25:277-286

23. Schwartz SA, Taljanovic MS, Smyth S, et al. (2007) CT findings of rupture, impending rupture, and contained rupture of abdominal aortic aneurysms [review]. AJR Am J Roentgenol 188(1):W57-W62 